\title{
A Generalized Pólya's Urn with Graph Based Interactions
}

\author{
Michel Benaïm, ${ }^{1}$ Itai Benjamini, ${ }^{2}$ Jun Chen, ${ }^{2}$ Yuri Lima ${ }^{3}$ \\ ${ }^{1}$ Institut de Mathématiques, Université de Neuchâtel, 11 rue Émile Argand, 2000 \\ Neuchâtel, Suisse; e-mail: michel.benaim@unine.ch \\ ${ }^{2}$ Weizmann Institute of Science, Faculty of Mathematics and Computer Science, \\ 76100, Rehovot, Israel; e-mail: itai.benjamini@weizmann.ac.il; jun.chen \\ ${ }^{3}$ Department of Mathematics, University of Maryland, College Park, Maryland \\ 20742; e-mail: yurilima@gmail.com
}

Received 21 November 2012; accepted 29 August 2013

Published online 21 November 2013 in Wiley Online Library (wileyonlinelibrary.com).

DOI 10.1002/rsa.20523

\begin{abstract}
Given a finite connected graph $G$, place a bin at each vertex. Two bins are called a pair if they share an edge of $G$. At discrete times, a ball is added to each pair of bins. In a pair of bins, one of the bins gets the ball with probability proportional to its current number of balls raised by some fixed power $\alpha>0$. We characterize the limiting behavior of the proportion of balls in the bins.

The proof uses a dynamical approach to relate the proportion of balls to a vector field. Our main result is that the limit set of the proportion of balls is contained in the equilibria set of the vector field. We also prove that if $\alpha<1$ then there is a single point $v=v(G, \alpha)$ with non-zero entries such that the proportion converges to $v$ almost surely.

A special case is when $G$ is regular and $\alpha \leq 1$. We show e.g. that if $G$ is non-bipartite then the proportion of balls in the bins converges to the uniform measure almost surely. (c) 2013 Wiley Periodicals, Inc. Random Struct. Alg., 46, 614-634, 2015

Keywords: gradient-like system; Pólya's urn; reinforcement; stochastic approximation algorithms; unstable equilibria
\end{abstract}

\section{INTRODUCTION AND STATEMENT OF RESULTS}

Let $G=(V, E)$ be a finite connected graph with $V=[m]=\{1, \ldots, m\}$ and $|E|=N$, and assume that on each vertex $i$ there is a bin initially with $B_{i}(0) \geq 1$ balls. For a fixed parameter $\alpha>0$, consider a random process of adding $N$ balls to these bins at each step, according to the following law: if the numbers of balls after step $n-1$ are $B_{1}(n-1), \ldots, B_{m}(n-1)$, step

Correspondence to: Y. Lima

(C) 2013 Wiley Periodicals, Inc. 
$n$ consists of adding, to each edge $\{i, j\} \in E$, one ball either to $i$ or to $j$, and the probability that the ball is added to $i$ is

$$
\mathbb{P}[i \text { is chosen among }\{i, j\} \text { at step } n]=\frac{B_{i}(n-1)^{\alpha}}{B_{i}(n-1)^{\alpha}+B_{j}(n-1)^{\alpha}} .
$$

In this paper, we study the limiting behavior, as the number of steps grows, of the proportion of balls in the bins of $G$. More specifically, let $N_{0}=\sum_{i=1}^{m} B_{i}(0)$ denote the initial total number of balls, let

$$
x_{i}(n)=\frac{B_{i}(n)}{N_{0}+n N}, \quad i \in[m]
$$

be the proportion of balls at vertex $i$ after step $n$, and let $x(n)=\left(x_{1}(n), \ldots, x_{m}(n)\right)$. Call the point $(1 / m, \ldots, 1 / m)$ the uniform measure. The first result classifies the limiting behavior of $x(n)$ when $G$ is regular and $\alpha=1$.

Theorem 1.1. Let $G$ be a finite, regular, connected graph, and let $\alpha=1$.

a. If $G$ is non-bipartite, then $x(n)$ converges to the uniform measure almost surely.

$b$. If $G$ is bipartite, then $x(n)$ converges to $\Omega$ almost surely.

Above, $\Omega$ is a subset of the $(m-1)$-dimensional closed simplex defined as follows: if $V=A \cup B$ is the bipartition of $G$, then

$$
\Omega=\left\{\left(x_{1}, \ldots, x_{m}\right): \exists p, q \geq 0, p+q=2 / m, \text { s.t. } x_{i}=p \text { on } A, x_{i}=q \text { on } B\right\} .
$$

Theorem 1.1 includes the case of any finite complete graph. A complete graph with at least three vertices is non-bipartite, thus the proportion of balls in the bins converges to the uniform measure almost surely.

Theorem 1.1 also includes the case of cycles: if the length is odd, then the proportion converges almost surely to the uniform measure; if the length is even, then the random process' limit set is contained in $\Omega$ almost surely.

Theorem 1.1 is consequence, after finer analysis, of a general result for any $G$ and any $\alpha>0$. We show that the random process is a stochastic approximation algorithm, thus it is related to a vector field in the closed simplex, and the limit set of the random process is contained in the equilibria set of the vector field (see Theorem 3.3). Let $\Lambda$ denote such an equilibria set.

Theorem 1.2. Let $G$ be a finite, connected graph, and let $\alpha>0$. Then the limit set of $x(n)$ is contained in $\Lambda$ almost surely.

Call $G$ balanced bipartite if there is a bipartition $V=A \cup B$ with $\# A=\# B$.

Corollary 1.3. Let $G$ be a finite, connected, not balanced bipartite graph, and let $\alpha=1$. Then $\Lambda$ is finite and $x(n)$ converges to an element of $\Lambda$ almost surely.

Theorem 1.2 also allows to characterize the limiting behavior of the random process when $\alpha<1$ and $G$ is any graph. 
Theorem 1.4. Let $G$ be a finite, connected graph, and let $\alpha<1$. Then there is $v=v(G, \alpha)$ with non-zero entries such that $x(n)$ converges to $v$ almost surely.

The last result deals with star graphs. The star graph is a tree with $m$ vertices and $m-1$ leaves.

Theorem 1.5. Let $G$ be a finite star graph with at least three vertices, and let $m$ be the vertex of highest degree.

a. If $\alpha \leq 1$, then $x(n)$ converges to

$$
\left(\frac{1}{m-1+(m-1)^{\frac{1}{1-\alpha}}}, \cdots, \frac{1}{m-1+(m-1)^{\frac{1}{1-\alpha}}}, \frac{(m-1)^{\frac{1}{1-\alpha}}}{m-1+(m-1)^{\frac{1}{1-\alpha}}}\right)
$$

almost surely.

b. If $\alpha>1$, then with positive probability $x(n)$ converges to $(0, \ldots, 0,1)$, and with positive probability $x(n)$ converges to $\left(\frac{1}{m-1}, \ldots, \frac{1}{m-1}, 0\right)$.

A motivation for the model proposed above is: imagine there are 3 companies, denoted by M, A and G. Each company sells two products. M sells OS and SE, A sells OS and SP, G sells SE and SP. Each pair of companies compete on one product. The companies try to use their global size and reputation to boost sales. A natural question is which company will sell more products in the long term. In this scenario, one can easily see that the interacting relation among the three companies forms a triangular network. On this triangle, a vertex represents a company and an edge represents a product. Under further simplifications, our model describes in broad strokes the long-term evolution of such competition.

Another motivation for the model comes from a repeated game in which agents improve their skill by gaining experience. The interaction network between agents is modeled by a graph. At each round a pair is competing for a ball. A competitor improves his skill with time, and the number of balls in his bin represents his skill level.

There are several natural ways to generalize our model to capture more complex interactions, but here we focus on the simplest setup. The model can be viewed as a class of graph based evolutionary model, which has been studied in various fields, e.g. biology [15], economics [9], mathematics [20], and sociology [19].

The classical Pólya's urn contains balls of two colors, and at each step one ball is drawn randomly, its color is observed, and a ball of the same color is added to the urn. In our process the interactions occur among pairs of bins, and on each of them the model evolves similar to the classical Pólya's urn. We therefore call it a generalized Pólya's urn with graph based interactions. For other variations on Pólya's urn, see e.g. $[6,12,14]$.

Let us sketch the ideas used in this article. We show that the proportion of balls in the bins is a stochastic approximation algorithm, then it is related to a vector field in the closed simplex. In our case the vector field is gradient-like: it has a strict Lyapunov function (see Definition 3.2), whose set of critical values has empty interior. This is enough to prove Theorem 1.2. Corollary 1.3 follows from concavity properties of the strict Lyapunov function.

The method described above is usually called a dynamical approach, because the vector field generates an autonomous ordinary differential equation (ODE), and dynamical information on the ODE give information on the random process. From now on, we make no distinction between the vector field and its ODE. 
The dynamical approach restricts the possible limits of the random process. Some equilibria are stable, and some are unstable. In order to exclude convergence to unstable equilibria, we look at the random process itself. Using probabilistic arguments, we give checkable conditions to guarantee that the random process has zero probability to converge to unstable equilibria (see Lemma 5.2). This is enough to prove Theorems 1.1, 1.4 and 1.5.

This work initiates a program to understand the limiting behavior of $x(n)$ and its dependence on $G$ and $\alpha$. Several open problems are presented in the last section.

\section{STOCHASTIC APPROXIMATION ALGORITHMS}

A stochastic approximation algorithm is a discrete time stochastic process whose general form can be written as

$$
x(n+1)-x(n)=\gamma_{n} H(x(n), \xi(n))
$$

where $H: \mathbb{R}^{m} \times \mathbb{R}^{m} \rightarrow \mathbb{R}^{m}$ is a measurable function that characterizes the algorithm, $\{x(n)\}_{n \geq 0} \subset \mathbb{R}^{m}$ is the sequence of parameters to be recursively updated, $\{\xi(n)\}_{n \geq 0} \subset \mathbb{R}^{m}$ is a sequence of random inputs where $H(x(n), \xi(n))$ is observable, and $\left\{\gamma_{n}\right\}_{n \geq 0}$ is a sequence of "small" nonnegative scalar gains. Such processes were first introduced in the early 50s on the works of Robbins and Monro [18] and Kiefer and Wolfowitz [10].

Let us show that the random process defined by (1.2) is a stochastic approximation algorithm. We start by specifically formulating the model. Write $i \sim j$ whenever $\{i, j\} \in E$. Let $\mathcal{F}_{n}$ denote the sigma-algebra generated by the process up to step $n$, and let $C_{i}(n)$ denote the number of balls added to $i$ at step $n$. For each neighboring pair of vertices $i \sim j$, consider 0 -1 valued random variables $\delta_{i \leftarrow j}(n+1), \delta_{j \leftarrow i}(n+1)$ such that $\delta_{i \leftarrow j}(n+1)+\delta_{j \leftarrow i}(n+1)=1$ and

$$
\mathbb{E}\left[\delta_{i \leftarrow j}(n+1) \mid \mathcal{F}_{n}\right]=\frac{B_{i}(n)^{\alpha}}{B_{i}(n)^{\alpha}+B_{j}(n)^{\alpha}}=\frac{x_{i}(n)^{\alpha}}{x_{i}(n)^{\alpha}+x_{j}(n)^{\alpha}} .
$$

Also, assume that $\delta_{i \leftarrow j}(n+1)$ and $\delta_{i^{\prime} \leftarrow j^{\prime}}(n+1)$ are independent whenever the edges $\{i, j\}$ and $\left\{i^{\prime}, j^{\prime}\right\}$ are distinct. Thus

$$
C_{i}(n+1)=\sum_{j \sim i} \delta_{i \leftarrow j}(n+1) .
$$

We want to show that $x(n)=\left(x_{1}(n), \ldots, x_{m}(n)\right)$ satisfies a difference equation of the form (2.1). Observe that

$$
\begin{aligned}
x_{i}(n+1)-x_{i}(n) & =\frac{B_{i}(n)+C_{i}(n+1)}{N_{0}+(n+1) N}-\frac{B_{i}(n)}{N_{0}+n N} \\
& =\frac{-N x_{i}(n)+C_{i}(n+1)}{N_{0}+(n+1) N} \\
& =\frac{1}{\frac{N_{0}}{N}+(n+1)}\left(-x_{i}(n)+\frac{1}{N} C_{i}(n+1)\right)
\end{aligned}
$$

and so $x(n)$ satisfies (2.1) with

$$
\gamma_{n}=\frac{1}{\frac{N_{0}}{N}+(n+1)}, \quad \xi(n)=\frac{1}{N}\left(C_{1}(n+1), \ldots, C_{m}(n+1)\right)
$$


and $H: \mathbb{R}^{m} \times \mathbb{R}^{m} \rightarrow \mathbb{R}^{m}$ defined by

$$
H(x(n), \xi(n))=-x(n)+\xi(n) .
$$

Conditioning on $\mathcal{F}_{n}, H$ has a deterministic component $x(n)$ and a random component $\xi(n)$. Nevertheless, nothing can be said about converging properties of $\xi(n)$. To this matter, we modify the above equation by decoupling $\xi(n)$ into its mean part and the so called "noise" part, which has zero mean. If one manages to control the total error of the noise term, the limiting behavior of $x(n)$ can be identified via the limiting behavior of the new deterministic component.

\section{THE DYNAMICAL APPROACH}

The dynamical approach is a method introduced by Ljung [13] and Kushner and Clark [11] to analyze stochastic approximation algorithms. Formally, it says that recursive expressions of the form (2.1) can be analyzed via an autonomous ODE

$$
\frac{d x(t)}{d t}=\bar{H}(x(t)),
$$

where $\bar{H}(x)=\lim _{n \rightarrow \infty} \mathbb{E}[H(x, \xi(n))]$.

In this perspective, our stochastic approximation algorithm can be written as

$$
x(n+1)-x(n)=\gamma_{n}\left\{\left(-x(n)+\mathbb{E}\left[\xi(n) \mid \mathcal{F}_{n}\right]\right)+\left(\xi(n)-\mathbb{E}\left[\xi(n) \mid \mathcal{F}_{n}\right]\right)\right\} .
$$

Denote $\xi(n)=\left(\xi_{1}(n), \ldots, \xi_{m}(n)\right)$. Eqs. (2.2), (2.3) and (2.4) imply

$$
\mathbb{E}\left[\xi_{i}(n) \mid \mathcal{F}_{n}\right]=\frac{1}{N} \sum_{j \sim i} \mathbb{E}\left[\delta_{i \leftarrow j}(n+1) \mid \mathcal{F}_{n}\right]=\frac{1}{N} \sum_{j \sim i} \frac{x_{i}(n)^{\alpha}}{x_{i}(n)^{\alpha}+x_{j}(n)^{\alpha}} .
$$

Thus, defining $\left\{u_{n}\right\}_{n \geq 0} \subset \mathbb{R}^{m}$ by

$$
u_{n}=\xi(n)-\mathbb{E}\left[\xi(n) \mid \mathcal{F}_{n}\right]
$$

and $F=\left(F_{1}, \ldots, F_{m}\right)$ by

$$
F_{i}\left(x_{1}, \ldots, x_{m}\right)=-x_{i}+\frac{1}{N} \sum_{j \sim i} \frac{x_{i}(n)^{\alpha}}{x_{i}(n)^{\alpha}+x_{j}(n)^{\alpha}},
$$

our random process takes the form

$$
x(n+1)-x(n)=\gamma_{n}\left[F(x(n))+u_{n}\right] .
$$

The above expression is a particular case of a class of stochastic approximation algorithms studied in [2], on which the behavior of the algorithm is related to a weak notion of recurrence for the ODE: that of chain-recurrence. Under the assumptions of Kushner and Clark lemma [11], it is proved that the accumulation points of $\{x(n)\}_{n \geq 0}$ are contained in the chain-recurrent set of the ODE. 
If, furthermore, the system is gradient-like and the set of critical values of the strict Lyapunov function has empty interior, then the accumulation points of $\{x(n)\}_{n \geq 0}$ are equilibria of the ODE. See Theorem 3.3 below.

In this section we introduce some definitions, state Theorem 3.3, and prove that our model satisfies two of the three conditions of Theorem 3.3.

Let $U \subset \mathbb{R}^{m}$ be a closed set, and let $F: U \rightarrow \mathbb{R}^{m}$ be a continuous vector field with unique integral curves.

Definition 3.1 (Equilibrium point). A point $x \in U$ is called an equilibrium if $F(x)=0$. $x$ is called stable if all the eigenvalues of $J F(x)$ have negative real part, and it is called unstable if one of the eigenvalues of $J F(x)$ has positive real part. Let $\Lambda$ denote the set of all equilibrium points. We call it the equilibria set.

Definition 3.2 (Strict Lyapunov function). A strict Lyapunov function for $F$ is a continuous map $L: U \rightarrow \mathbb{R}$ which is strictly monotone along any integral curve of $F$ outside $\Lambda$. In this case, we call $F$ gradient-like.

\subsection{A Limit Set Theorem}

The reason we can characterize the limit set of the random process via the equilibria set of the vector field is due to results in [2-4] which, to our purposes, are summarized as follows.

Theorem 3.3. Let $F: \mathbb{R}^{m} \rightarrow \mathbb{R}^{m}$ be a continuous gradient-like vector field with unique integral curves, let $\Lambda$ be its equilibria set, let $L$ be a strict Lyapunov function, and let $\{x(n)\}_{n \geq 0}$ be a solution to the recursion

$$
x(n+1)-x(n)=\gamma_{n}\left[F(x(n))+u_{n}\right]
$$

where $\left\{\gamma_{n}\right\}_{n \geq 0}$ is a decreasing gain sequence $e^{l}$ and $\left\{u_{n}\right\}_{n \geq 0} \subset \mathbb{R}^{m}$. Assume that

1. $\{x(n)\}_{n \geq 0}$ is bounded,

2. for each $T>0$,

$$
\lim _{n \rightarrow \infty}\left(\sup _{\left\{k: 0 \leq \tau_{k}-\tau_{n} \leq T\right\}}\left\|\sum_{i=n}^{k-1} \gamma_{i} u_{i}\right\|\right)=0,
$$

where $\tau_{n}=\sum_{i=0}^{n-1} \gamma_{i}$, and

3. $L(\Lambda) \subset \mathbb{R}$ has empty interior.

Then the limit set of $\{x(n)\}_{n \geq 0}$ is a connected subset of $\Lambda$.

Proof. See Theorem 1.2 of [2] and Proposition 6.4 of [3].

${ }^{1} \lim _{n \rightarrow \infty} \gamma_{n}=0$ and $\sum_{n \geq 0} \gamma_{n}=\infty$. 


\subsection{The Random Process (3.4) Satisfies 1 and 2 of Theorem 3.3}

Firstly, note that $\left\{\gamma_{n}\right\}_{n \geq 0}$ satisfies

$$
\lim _{n \rightarrow \infty} \gamma_{n}=0 \text { and } \sum_{n \geq 0} \gamma_{n}=\infty
$$

Of course, $\{x(n)\}_{n \geq 0}$ is bounded. It remains to check condition 2. For that, let

$$
M_{n}=\sum_{i=0}^{n-1} \gamma_{i} u_{i}
$$

$\left\{M_{n}\right\}_{n \geq 0}$ is a martingale adapted to the filtration $\left\{\mathcal{F}_{n}\right\}_{n \geq 0}$ :

$$
\mathbb{E}\left[M_{n+1} \mid \mathcal{F}_{n}\right]=\sum_{i=0}^{n-1} \gamma_{i} u_{i}+\mathbb{E}\left[\gamma_{n+1} u_{n+1} \mid \mathcal{F}_{n}\right]=\sum_{i=0}^{n-1} \gamma_{i} u_{i}=M_{n}
$$

Furthermore, because for any $n \geq 0$

$$
\sum_{i=0}^{n} \mathbb{E}\left[\left\|M_{i+1}-M_{i}\right\|^{2} \mid \mathcal{F}_{i}\right] \leq \sum_{i=0}^{n} \gamma_{i}^{2} \leq \sum_{i \geq 0} \gamma_{i}^{2}<\infty \text { a.s. }
$$

the sequence $\left\{M_{n}\right\}_{n \geq 0}$ converges almost surely to a finite random vector (see e.g. Theorem 5.4.9 of [7]). In particular, it is a Cauchy sequence and so condition 2 holds almost surely.

In order to apply Theorem 3.3, we will construct a strict Lyapunov function for the ODE

$$
\left\{\begin{array}{cc}
\frac{d v_{1}(t)}{d t} & =-v_{1}(t)+\frac{1}{N} \sum_{j \sim 1} \frac{v_{1}(t)^{\alpha}}{v_{1}(t)^{\alpha}+v_{j}(t)^{\alpha}} \\
\frac{d v_{m}(t)}{d t} & =-v_{m}(t)+\frac{1}{N} \sum_{j \sim m} \frac{v_{m}(t)^{\alpha}}{v_{m}(t)^{\alpha}+v_{j}(t)^{\alpha}}
\end{array}\right.
$$

that satisfies condition (iii) of Theorem 3.3.

Before that, let us specify the domain of the vector field $F$. Fix $c<1 / N$, and let $\Delta$ be the set of $m$-tuples $\left(x_{1}, \ldots, x_{m}\right) \in \mathbb{R}^{m}$ such that:

1. $x_{i} \geq 0$ and $\sum_{i=1}^{m} x_{i}=1$, and

2. $x_{i}+x_{j} \geq c$ for all $\{i, j\} \in E$.

Clearly, $F: \Delta \rightarrow T \Delta$ is Lipschitz. Moreover, we have

Lemma 3.4. $\Delta$ is positively invariant under the ODE (3.5). 
Proof. Given $\{i, j\} \in E$,

$$
\begin{aligned}
\frac{d}{d t}\left(v_{i}+v_{j}\right) & =-v_{i}+\frac{1}{N} \sum_{k \sim i} \frac{v_{i}^{\alpha}}{v_{i}^{\alpha}+v_{k}^{\alpha}}-v_{j}+\frac{1}{N} \sum_{l \sim j} \frac{v_{j}^{\alpha}}{v_{j}^{\alpha}+v_{l}^{\alpha}} \\
& \geq-\left(v_{i}+v_{j}\right)+\frac{1}{N}\left(\frac{v_{i}^{\alpha}}{v_{i}^{\alpha}+v_{j}^{\alpha}}+\frac{v_{j}^{\alpha}}{v_{j}^{\alpha}+v_{i}^{\alpha}}\right) \\
& =-\left(v_{i}+v_{j}\right)+\frac{1}{N} .
\end{aligned}
$$

If $v$ belongs to the boundary of $\Delta$, there exists some $\{i, j\} \in E$ such that $v_{i}+v_{j}=c$, and then

$$
\frac{d}{d t}\left(v_{i}+v_{j}\right) \geq-\left(v_{i}+v_{j}\right)+\frac{1}{N}=-c+\frac{1}{N}>0,
$$

which means that $F$ points inward on the boundary of $\Delta$. This proves that condition 2 is preserved.

\section{THE GENERAL CASE: PROOF OF THEOREM 1.2}

Let $L: \Delta \rightarrow \mathbb{R}$ be given by

$$
L\left(v_{1}, \ldots, v_{m}\right)=-\sum_{i=1}^{m} v_{i}+\frac{1}{\alpha N} \sum_{\{i, j\} \in E} \log \left(v_{i}^{\alpha}+v_{j}^{\alpha}\right) .
$$

Lemma 4.1. L is a strict Lyapunov function for $F$.

Proof. We have

$$
\frac{\partial L}{\partial v_{i}}=-1+\frac{1}{\alpha N} \sum_{i \sim j} \frac{\alpha v_{i}^{\alpha-1}}{v_{i}^{\alpha}+v_{j}^{\alpha}}=-1+\frac{1}{N} \sum_{i \sim j} \frac{v_{i}^{\alpha-1}}{v_{i}^{\alpha}+v_{j}^{\alpha}},
$$

thus

$$
\frac{d v_{i}}{d t}=v_{i}\left(-1+\frac{1}{N} \sum_{i \sim j} \frac{v_{i}^{\alpha-1}}{v_{i}^{\alpha}+v_{j}^{\alpha}}\right)=v_{i} \frac{\partial L}{\partial v_{i}} .
$$

If $v=\left(v_{1}(t), \ldots, v_{m}(t)\right), t \geq 0$, is an integral curve of $F$, then (4.3) implies

$$
\frac{d}{d t}(L \circ v)=\sum_{i=1}^{m} \frac{\partial L}{\partial v_{i}} \frac{d v_{i}}{d t}=\sum_{i=1}^{m} v_{i}\left(\frac{\partial L}{\partial v_{i}}\right)^{2} \geq 0 .
$$

Also, the last expression is zero if and only if $v_{i}\left(\frac{\partial L}{\partial v_{i}}\right)^{2}=0$ for all $i \in[m]$, which is equivalent to $F(v)=0$.

Let $\Lambda \subset \Delta$ be the equilibria set of $F$. For each $S \subset[m]$, let

$$
\Delta_{S}=\left\{v \in \Delta: v_{i}=0 \text { iff } i \notin S\right\}
$$


denote the face of $\Delta$ determined by $S . \Delta_{S}$ is a manifold with corners, positively invariant under the ODE (3.5).

Definition 4.2. $v \in \Delta_{S}$ is an $S$-singularity for $L$ if

$$
\frac{\partial L}{\partial v_{i}}(v)=0 \text { for all } i \in S
$$

Let $\Lambda_{S} \subset \Delta_{S}$ denote the set of $S$-singularities for $L$.

Lemma 4.3. $\Lambda=\bigcup_{S \subset[m]} \Lambda_{S}$.

Proof. If $v \in \Delta_{S}$, then $\frac{d v_{i}}{d t}=v_{i} \frac{\partial L}{\partial v_{i}}=0$ for all $i \notin S$. Thus $v \in \Lambda$ iff $\frac{\partial L}{\partial v_{i}}=0$ for all $i \in S$.

To prove Theorem 1.2, it remains to show that $L(\Lambda)$ has empty interior. $\left.L\right|_{\Delta_{S}}$ is a $C^{\infty}$ function, thus by Sard's theorem $L\left(\Lambda_{S}\right)$ has zero Lebesgue measure, so $L(\Lambda)$ has zero Lebesgue measure as well. In particular, it has empty interior.

\subsection{Proof of Corollary 1.3}

Each restriction $\left.L\right|_{\Delta_{S}}$ is concave ${ }^{2}$. We claim that $\left.L\right|_{\Delta_{S}}$ is strictly concave. Let $u, v \in \Delta_{S}$ and $c \in(0,1)$. If $L(c u+(1-c) v)=c L(u)+(1-c) L(v)$, then $u_{i}+u_{j}=v_{i}+v_{j}$ for every $\{i, j\} \in E$, i.e.

$$
u_{i}-v_{i}=(-1)\left(u_{j}-v_{j}\right), \quad \forall\{i, j\} \in E .
$$

The values of $u_{i}-v_{i}$ along any path in $G$ alternate between $u_{1}-v_{1}$ and $-\left(u_{1}-v_{1}\right)$ :

$$
u_{i}-v_{i}= \begin{cases}u_{1}-v_{1} & \text { if the distance from } i \text { to } 1 \text { is even } \\ -\left(u_{1}-v_{1}\right) & \text { if the distance from } i \text { to } 1 \text { is odd }\end{cases}
$$

Let $A$ be the vertices within even distance to 1 and $B$ those within odd distance to 1 . If $G$ is non-bipartite, then $A \cap B \neq \varnothing$, thus $u_{1}=v_{1}$. If $G$ is bipartite, then $V=A \cup B$ is the bipartition. By (4.4),

$$
0=\sum_{i=1}^{m} u_{i}-\sum_{i=1}^{m} v_{i}=\left(u_{1}-v_{1}\right) \# A-\left(u_{1}-v_{1}\right) \# B .
$$

Because $G$ is not balanced bipartite, $\# A \neq \# B$ and again $u_{1}=v_{1}$. In any case we have $u_{1}=v_{1}$, i.e. $u=v$. Thus $\left.L\right|_{\Delta_{S}}$ is strictly concave. By the same reasoning as in the end the proof of Theorem 1.2, the corollary is established.

${ }^{2}$ This follows from a simple fact on convex functions. Let $\psi: \mathbb{R} \rightarrow \mathbb{R}$ be an increasing, concave function, and let $f: \mathbb{R}^{k} \rightarrow \mathbb{R}$ be a (strictly) concave function. Then $\psi \circ f$ is also (strictly) concave. 


\section{NON-CONVERGENCE TO UNSTABLE EQUILIBRIA}

In this section we give checkable conditions to guarantee that the random process has zero probability to converge to unstable equilibria of $F$ when $\alpha \leq 1$. This is Lemma 5.2, and its proof is an adaptation of the proof of Theorem 1.3 in [16].

The checkable condition is in terms of the partial derivatives of $L$. Before stating it, let us make some general considerations. By (4.2),

$$
\frac{\partial L}{\partial v_{1}}=-1+\frac{1}{N} \sum_{i \sim 1} \frac{v_{1}^{\alpha-1}}{v_{1}^{\alpha}+v_{i}^{\alpha}} .
$$

$\partial L / \partial v_{1}$ is finite if $\alpha=1$ or $v_{1}>0$, and infinite otherwise. Define $\partial L / \partial v_{1}: \Delta \rightarrow \mathbb{R} \cup\{\infty\}$, which is continuous. In particular, if $\partial L / \partial v_{1}(v)>0$ then it is positive in a neighborhood of $v$. In the proof of Lemma 5.2 we will make use of the lemma below.

Lemma 5.1. Let $v \in \Delta$ with $v_{1}=0$ and $\partial L / \partial v_{1}(v)>3 \delta$. Then there exists a neighborhood $\mathcal{N}$ of $v$, an element $u \in \mathcal{N}$, and $\varepsilon_{0}>0$ such that

1. $\frac{\partial L}{\partial v_{1}}(u)>3 \delta+\frac{m \varepsilon_{0}}{N}$, and

2. for all $w \in \mathcal{N}$ and $i \sim 1$ it holds

$$
\frac{w_{1}^{\alpha-1}}{w_{1}^{\alpha}+w_{i}^{\alpha}}>\frac{u_{1}^{\alpha-1}}{u_{1}^{\alpha}+u_{i}^{\alpha}}-\varepsilon_{0} .
$$

Proof. Firstly, assume that $\alpha=1 . \partial L / \partial v_{1}$ is continuous, so we can fix a neighborhood $\mathcal{N}$ of $v$ satisfying condition 1 . Each $w \in \overline{\mathcal{N}} \mapsto 1 /\left(w_{1}+w_{i}\right), i \sim 1$, is uniformly continuous, thus condition 2 also holds if $\mathcal{N}$ is small enough.

Now assume that $\alpha<1$. Again, we can fix a neighborhood $\mathcal{N}$ of $v$ satisfying condition 1. In this case, the maps $L_{i}: w \in \overline{\mathcal{N}} \mapsto w_{1}^{\alpha-1} /\left(w_{1}^{\alpha}+w_{i}^{\alpha}\right), i \sim 1$, are not uniformly continuous. But they are convex ${ }^{3}$. Because $\left.L_{i}\right|_{\overline{\mathcal{N}} \cap\left\{w_{1}=0\right\}}=\infty$, its minimum is attained outside $\overline{\mathcal{N}} \cap\left\{w_{1}=0\right\}$. Thus we can choose a small neighborhood $\mathcal{V}$ of $\overline{\mathcal{N}} \cap\left\{w_{1}=0\right\}$ that does not contain the minima of none of the $L_{i}$. Now, $\left.L_{i}\right|_{\mathcal{N} \backslash \mathcal{V}}$ is uniformly continuous. Restrict $\mathcal{N}$ if necessary and choose any $u \in \overline{\mathcal{N}} \backslash \mathcal{V}$. Thus if $w \in \overline{\mathcal{N}} \backslash \mathcal{V}$ then $L_{i}(w)>L_{i}(u)-\varepsilon_{0}$, and if $w \in \mathcal{V}$ then $L_{i}(w) \geq\left.\min L_{i}\right|_{\overline{\mathcal{N}} \backslash \mathcal{V}}>L_{i}(u)-\varepsilon_{0}$.

Lemma 5.2. Let $G$ be a finite, connected graph, $\alpha \leq 1$ and $L$ as in (4.1). Let $v$ be an equilibrium with $v_{1}=0$. If $\partial L / \partial v_{1}(v)>0$, then

$$
\mathbb{P}\left[\lim _{n \rightarrow \infty} x(n)=v\right]=0 .
$$

Before embarking into the proof, let us explain why the conditions of the lemma are equivalent to $v$ being an unstable equilibrium. Firstly, assume that $\alpha=1$. We look at the jacobian matrix $J F(v)$ :

${ }^{3}$ This is consequence of two facts of convex functions. Fact 1. If $f: \mathbb{R}^{k} \rightarrow(0, \infty)$ is concave, then $1 / f$ is convex. Fact 2. $(x, y) \in(0, \infty)^{2} \mapsto x^{\alpha} y^{1-\alpha}$ is concave. 


$$
\frac{\partial F_{i}}{\partial v_{j}}= \begin{cases}v_{i} \frac{\partial^{2} L}{\partial v_{i} \partial v_{j}} & \text { if } i \sim j \\ \frac{\partial L}{\partial v_{i}}+v_{i} \frac{\partial^{2} L}{\partial v_{i}^{2}} & \text { if } i=j \\ 0 & \text { otherwise. }\end{cases}
$$

Without loss of generality, assume that $v \in \Delta_{S}$ with $S=\{k+1, \ldots, m\}$. Thus

$$
J F(v)=\left[\begin{array}{ll}
A & 0 \\
C & B
\end{array}\right]
$$

where $A$ is a $k \times k$ diagonal matrix with $a_{i i}=\partial L / \partial v_{i}, i \in[k]$. The spectrum of $J F(v)$ is the union of the spectra of $A$ and $B$. With respect to the inner product $(x, y)=\sum_{i=k+1}^{m} x_{i} y_{i} / v_{i}$, $B$ is self-adjoint and negative semidefinite (by the concavity of $L$ ), hence the eigenvalues of $B$ are real and nonpositive. Therefore, $J F(v)$ has one real positive eigenvalue if and only if one of $a_{i i}$ is positive.

When $\alpha<1, J F(v)$ is not defined, in particular because $a_{11}$ is not finite. Nevertheless, $a_{11}$ explodes to infinity, which intuitively means that $v$ is unstable.

Proof. Firstly, we claim that

$$
\mathbb{P}\left[\lim _{n \rightarrow \infty} B_{1}(n)=\infty\right]=1 .
$$

This is easy: because $1 \leq B_{i}(n) \leq N_{0}+n N$, we have

$$
\mathbb{P}[1 \text { is chosen among }\{1, i\} \text { at step } n+1]=\frac{B_{1}(n)^{\alpha}}{B_{1}(n)^{\alpha}+B_{i}(n)^{\alpha}} \geq \frac{1}{2\left(N_{0}+n N\right)}
$$

for every $i \sim 1$, and so (5.3) follows from the Borel-Cantelli lemma.

Fix $B>0$ large enough (to be specified later), and define

$$
\mathcal{Y}_{n}=\{x(k) \in \mathcal{N}, \forall k \geq n\} \cap\left\{B_{1}(n)>B\right\}, n>0 .
$$

By (5.3), $\left\{\lim _{n \rightarrow \infty} x(n)=v\right\} \subset \bigcup_{m \geq n} \mathcal{Y}_{m}$ for any $n>0$. Thus the lemma will be proved if we show that

$$
\mathbb{P}\left[\mathcal{Y}_{n}\right]=0 \text { for sufficiently large } n .
$$

Let $\delta>0$ and $\mathcal{N}$ as in Lemma 5.1. For a fixed $n_{0}$, let $\mathcal{G}_{n}=\mathcal{F}_{n} \cap \mathcal{Y}_{n_{0}}$, and let $c>0$ such that

$$
\left[1+\frac{\delta(1+2 \delta)}{1+\frac{3}{2} \delta}\right] \frac{1}{1+\delta}=1+c .
$$

We claim that if $B$ is large enough, then there is $n_{0}>0$ such that

$$
\mathbb{E}\left[\log x_{1}((1+\delta) n) \mid \mathcal{G}_{n}\right] \geq \log x_{1}(n)+\frac{1}{2} \log (1+c) \text { for all } n>n_{0} .
$$


Before proving the claim, let us show how to conclude the proof of the lemma. By contradiction, assume that (5.4) is not true for some $n>n_{0}$. Define $T_{k}=(1+\delta)^{k} n$ and $X_{k}=\log x_{1}\left(T_{k}\right)$. By (5.6),

$$
\mathbb{E}\left[X_{k+1} \mid \mathcal{G}_{n}\right]=\mathbb{E}\left[\mathbb{E}\left[X_{k+1} \mid \mathcal{G}_{T_{k}}\right] \mid \mathcal{G}_{n}\right] \geq \mathbb{E}\left[X_{k} \mid \mathcal{G}_{n}\right]+\frac{1}{2} \log (1+c) .
$$

By induction,

$$
\mathbb{E}\left[X_{k} \mid \mathcal{G}_{n}\right] \geq X_{0}+\frac{k}{2} \log (1+c) \geq-\log \left(N_{0}+n N\right)+\frac{k}{2} \log (1+c)
$$

which is a contradiction, because the left hand side is bounded.

We now prove (5.6). The proof uses a coupling argument and Chernoff bounds. Let $t \in\{n+1, \ldots,(1+\delta) n\}$. Restricted to $\mathcal{Y}_{n_{0}}$, we have

$$
\begin{aligned}
\mathbb{P}[1 \text { is chosen among }\{1, i\} \text { at step } t] & =\frac{B_{1}(t-1)^{\alpha}}{B_{1}(t-1)^{\alpha}+B_{i}(t-1)^{\alpha}} \\
& \geq \frac{B_{1}(n)}{N_{0}+(t-1) N} \cdot \frac{x_{1}(t-1)^{\alpha-1}}{x_{1}(t-1)^{\alpha}+x_{i}(t-1)^{\alpha}} \\
& \geq \frac{B_{1}(n)}{N_{0}+(t-1) N}\left(\frac{u_{1}^{\alpha-1}}{u_{1}^{\alpha}+u_{i}^{\alpha}}-\varepsilon_{0}\right) .
\end{aligned}
$$

Define a family of independent Bernoulli random variables $\left\{E_{t, i}\right\}, t=n+1, \ldots,(1+\delta) n$, $i \sim 1$, as follows

$$
\mathbb{P}\left[E_{t, i}=1\right]=\frac{B_{1}(n)}{N_{0}+(t-1) N}\left(\frac{u_{1}^{\alpha-1}}{u_{1}^{\alpha}+u_{i}^{\alpha}}-\varepsilon_{0}\right) .
$$

Now couple $\left\{E_{t, i}\right\}$ to our model: if $E_{t, i}=1$, then 1 is chosen among $\{1, i\}$ at step $t$. If $n_{0}$ is large enough ${ }^{4}$, then

$$
\begin{aligned}
\mathbb{E}\left[\sum_{\substack{n+1 \leq t \leq(1+\delta) n \\
i \sim 1}} E_{t, i}\right] & \geq B_{1}(n)\left(\sum_{t=n+1}^{(1+\delta) n} \frac{1}{N_{0}+(t-1) N}\right)\left(\sum_{i \sim 1} \frac{u_{1}^{\alpha-1}}{u_{1}^{\alpha}+u_{i}^{\alpha}}-m \varepsilon_{0}\right) \\
& >B_{1}(n) \frac{\delta(1+3 \delta)}{1+\frac{3}{2} \delta} .
\end{aligned}
$$

By Chernoff bounds (see Corollary A.1.14 of [1]), if $\varepsilon_{1}>0$ then there is $B_{0}$ large enough such that

$$
\mathbb{P}\left[\sum_{\substack{n+1 \leq t \leq 1+\delta) n \\ i \sim 1}} E_{t, i}>B_{1}(n) \frac{\delta(1+2 \delta)}{1+\frac{3}{2} \delta}\right]>1-\varepsilon_{1}
$$

${ }^{4}$ Because $\log (1+x)>\frac{x}{1+x}$ for small $x>0$, if $n_{0}$ is large then

$$
N \sum_{t=n+1}^{(1+\delta) n} \frac{1}{N_{0}+(t-1) N}>\log \left(\frac{N_{0}+(1+\delta) n N}{N_{0}+n N}\right)>\log \left(1+\frac{\delta}{1+\frac{1}{2} \delta}\right)>\frac{\delta}{1+\frac{3}{2} \delta} .
$$

Random Structures and Algorithms DOI 10.1002/rsa 
for every $B_{1}(n)>B_{0}$. Whenever (5.7) holds, the coupling gives that

$$
B_{1}((1+\delta) n)-B_{1}(n) \geq \sum_{\substack{n+1 \leq t \leq 1 \\ i \sim 1 \\ i \sim \delta) n}} E_{t, i}>B_{1}(n) \frac{\delta(1+2 \delta)}{1+\frac{3}{2} \delta}
$$

and thus by (5.5) we have

$$
x_{1}((1+\delta) n)>x_{1}(n)\left[1+\frac{\delta(1+2 \delta)}{1+\frac{3}{2} \delta}\right] \frac{1}{1+\delta}=x_{1}(n)(1+c) .
$$

From (5.7), it follows that

$$
\mathbb{P}\left[x_{1}((1+\delta) n)>x_{1}(n)(1+c) \mid \mathcal{G}_{n}\right]>1-\varepsilon_{1} .
$$

Because $x_{1}((1+\delta) n)>\frac{x_{1}(n)}{1+\delta}$ and $\varepsilon_{1}>0$ can be chosen arbitrarily small, (5.9) gives

$$
\begin{aligned}
\mathbb{E}\left[\log x_{1}((1+\delta) n) \mid \mathcal{G}_{n}\right] & >\left(1-\varepsilon_{1}\right) \log \left(x_{1}(n)(1+c)\right)+\varepsilon_{1} \log \left(\frac{x_{1}(n)}{1+\delta}\right) \\
& >\log x_{1}(n)+\frac{1}{2} \log (1+c),
\end{aligned}
$$

thus establishing (5.6).

\section{REGULAR GRAPHS: PROOF OF THEOREM 1.1}

Here, $G$ is a finite, $r$-regular, connected graph, and $\alpha=1$. Assume first that $G$ is nonbipartite, and let $u \in \Delta$ be the uniform measure. By Corollary 1.3, $\Lambda$ is finite and $x(n)$ converges to an element of $\Lambda$. Furthermore, $\# \Lambda_{S} \leq 1$ for every $S \subset[m]$. It is easy to check that $u \in \Lambda_{[m]}$, thus $\Lambda_{[m]}=\{u\}$. We will show that any other equilibrium satisfies the conditions of Lemma 5.2, in which case we conclude the proof of Theorem 1.1 (a).

Now assume that $G$ is bipartite, and that $V=A \cup B$ is the bipartition of $G$. Let $\Omega$ be defined as in (1.3). Every $v \in \Omega$ is an equilibrium: for $i \in A$

$$
F_{i}(v)=-v_{i}+\frac{1}{N} \sum_{j \sim i} \frac{v_{i}}{v_{i}+v_{j}}=-p+\frac{r p}{N(p+q)}=0,
$$

and the same holds for $i \in B$. We will show that any other equilibrium satisfies the conditions of Lemma 5.2 and thus is unstable. This being proved, Theorem 1.1 (b) is established.

Summarizing the above discussion, to prove Theorem 1.1 we just need to prove the lemma below.

Lemma 6.1. Let $G$ be a finite, regular, connected graph, and let $\alpha=1$.

a. If $G$ is non-bipartite, then every element of $\Lambda \backslash\{u\}$ is unstable.

$b$. If $G$ is bipartite, then every element of $\Lambda \backslash \Omega$ is unstable.

Proof. Let $v \in \Lambda_{S}$ satisfying either (a) or (b). By Lemma 5.2, it is enough to show that $\partial L / \partial v_{i}>0$ for some $i \in[m] \backslash S$. Since $\partial L / \partial v_{i}=0$ for $i \in S$, it suffices to show that $\sum_{i=1}^{m} \frac{\partial L}{\partial v_{i}}>0$, i.e.

$$
-m+\frac{1}{N} \sum_{i=1}^{m} \sum_{j \sim i} \frac{1}{v_{i}+v_{j}}>0 .
$$


We first claim that the above expression is nonnegative. For this, note that the summand has $2 N$ terms and, by the arithmetic-harmonic mean inequality,

$$
\left[\sum_{i=1}^{m} \sum_{j \sim i} \frac{1}{v_{i}+v_{j}}\right] \cdot\left[\sum_{i=1}^{m} \sum_{j \sim i}\left(v_{i}+v_{j}\right)\right] \geq(2 N)^{2},
$$

with equality if and only if

$$
v_{i}+v_{j}=\text { const., } \forall\{i, j\} \in E .
$$

Since $G$ is $r$-regular, $N=r m / 2$ and

$$
\sum_{i=1}^{m} \sum_{j \sim i}\left(v_{i}+v_{j}\right)=2 r .
$$

So (6.2) gives

$$
\sum_{i=1}^{m} \sum_{j \sim i} \frac{1}{v_{i}+v_{j}} \geq \frac{(2 N)^{2}}{2 r}=N m
$$

thus proving our claim.

If (6.1) is not true, then (6.3) holds. Fix the vertex 1 of $G$, and let $v_{1}=p$ and $v_{i}=q$ for every neighbor $i \sim 1$. Thus the values of $v_{i}$ along any path in the graph $G$ alternate between $p$ and $q$, i.e.

$$
v_{i}= \begin{cases}p & \text { if the distance from } i \text { to } 1 \text { is even, } \\ q & \text { if the distance from } i \text { to } 1 \text { is odd. }\end{cases}
$$

If $G$ is non-bipartite, it has a cycle of odd length, then $p=q$ and $v=u$, and if $G$ is bipartite then $v \in \Omega$. In both cases, we get a contradiction.

\section{PROOF OF THEOREM 1.4}

If $\alpha<1$, then the function $x \mapsto x^{\alpha}, x>0$, is strictly concave. Thus each restriction $\left.L\right|_{\Delta_{S}}$ is also strictly concave ${ }^{5}$, so $L$ has at most one $S$-singularity.

If $S \neq[m]$, then $v_{i}=0$ and $\partial L / \partial v_{i}=\infty$ on $\Lambda_{S}$. By Lemma $5.2, \Lambda_{S}$ consists of an unstable equilibrium. Let $\Lambda_{[m]}=\{v\}$. Thus $v$ has non-zero entries and $x(n)$ converges to $v$ almost surely.

\section{STAR GRAPHS: PROOF OF THEOREM 1.5}

When $G$ is the star graph with $m$ vertices and $m$ is the vertex with higher degree, (3.5) becomes

$$
\left\{\begin{array}{l}
\frac{d v_{i}(t)}{d t}=-v_{i}(t)+\frac{1}{m-1} \cdot \frac{v_{i}(t)^{\alpha}}{v_{i}(t)^{\alpha}+v_{m}(t)^{\alpha}}, \quad i \in[m-1] \\
\frac{d v_{m}(t)}{d t}=-v_{m}(t)+\frac{1}{m-1} \sum_{j=1}^{m-1} \frac{v_{m}(t)^{\alpha}}{v_{m}(t)^{\alpha}+v_{j}(t)^{\alpha}}
\end{array}\right.
$$

\footnotetext{
${ }^{5}$ Again, we are using that if $\psi: \mathbb{R} \rightarrow \mathbb{R}$ is an increasing, concave function, and $f: \mathbb{R}^{k} \rightarrow \mathbb{R}$ is a (strictly) concave function, then $\psi \circ f$ is also (strictly) concave.
} 
Case 1. $\alpha \leq 1$.

When $\alpha=1$, note that $(0, \ldots, 1) \in \Lambda$. We will show that any $v \in \Lambda \backslash\{(0, \ldots, 0,1)\}$ satisfies the conditions of Lemma 5.2. By the arithmetic-harmonic mean inequality,

$$
\frac{\partial L}{\partial v_{m}}=-1+\frac{1}{m-1} \sum_{i=1}^{m-1} \frac{1}{v_{i}+v_{m}} \geq-1+\frac{m-1}{1+(m-2) v_{m}}>0 .
$$

Because $v \in \Lambda$, we also have $v_{m}=0$.

When $\alpha<1$, direct calculations show that

$$
\left(\frac{1}{m-1+(m-1)^{\frac{1}{1-\alpha}}}, \ldots, \frac{1}{m-1+(m-1)^{\frac{1}{1-\alpha}}}, \frac{(m-1)^{\frac{1}{1-\alpha}}}{m-1+(m-1)^{\frac{1}{1-\alpha}}}\right)
$$

is an equilibrium point in the interior of $\Delta$. By concavity of $L$, it is the unique equilibrium point in the interior of $\Delta$. The result thus follows from Theorem 1.4.

Case 2. $\alpha>1$.

When $m=2$, our model is a class of generalized Pólya's urn. For simplicity, we refer to this process as "g-urn". It is known (see e.g. Theorem 4.1 of [8]) that in this case

$$
\mathbb{P}\left[\lim _{n \rightarrow \infty} x(n)=(0,1)\right]>0 \text { and } \mathbb{P}\left[\lim _{n \rightarrow \infty} x(n)=(1,0)\right]>0 .
$$

Now assume $m>2$. Observe that, as events,

$$
\left\{\lim _{n \rightarrow \infty} x(n)=\left(\frac{1}{m-1}, \ldots, \frac{1}{m-1}, 0\right)\right\} \supset \bigcap_{i=1}^{m-1} \bigcap_{n \geq 1}\left\{\delta_{i \leftarrow m}(n)=1\right\} .
$$

By a coupling argument, we can identify this last event to the following one: in $m-1$ independent g-urns, just one color of ball is added in each g-urn since the beginning of the process. Rubin's Theorem (see e.g. Theorem 3.6 of [17]) guarantees that the event "just one color of ball is added to the g-urn since the beginning of the process" has positive probability, and so

$$
\mathbb{P}\left[\lim _{n \rightarrow \infty} x(n)=\left(\frac{1}{m-1}, \ldots, \frac{1}{m-1}, 0\right)\right]>0 .
$$

To prove the other claim, first observe that

$$
\left\{\lim _{n \rightarrow \infty} x(n)=(0, \ldots, 0,1)\right\} \supset \bigcap_{i=1}^{m-1} \bigcap_{n \geq 1}\left\{\delta_{i \leftarrow m}(n)=0\right\} .
$$

By a coupling argument, the term on the right hand side of the above inclusion has positive probability (again by Rubin's Theorem). This concludes the proof of Theorem 1.5.

Remark 8.1. Given a finite connected graph $G=(V, E)$, call $I \subset V$ an independent set if $\{i, j\} \notin E$ for $i, j \in I$. The proof of Theorem 1.5 gives the following: if $\alpha>1$ and $I$ is an independent set, then

$$
\mathbb{P}\left[\lim _{n \rightarrow \infty} x_{i}(n)=0, \forall i \in I\right]>0 .
$$




\section{VARIANTS OF THE MODEL}

\subsection{Edges with Different Weight Functions}

Let $G=(V, E)$ be a finite, connected graph. For each edge $\{i, j\} \in E$, let $f_{\{i, j\}}:(0,1) \rightarrow$ $(0,1)$. A variant of the model is described as follows. Let $x_{1}(n-1), \ldots, x_{m}(n-1)$ be the proportions of balls after step $n-1$. At step $n$, for each edge $\{i, j\} \in E$ add one ball either to $i$ or $j$ with probability

$$
\mathbb{P}[i \text { is chosen among }\{i, j\} \text { at step } n]=\frac{f_{\{i, j\}}\left(x_{i}(n-1)\right)}{f_{\{i, j\}}\left(x_{i}(n-1)\right)+f_{\{i, j\}}\left(x_{j}(n-1)\right)} .
$$

In other words, we replace the law of $\delta_{i \leftarrow j}(n)$ in (2.2) by the above one, defined in terms of the $f_{\{i, j\}}$ 's.

If we assume that, for each $\{i, j\} \in E, f_{\{i, j\}}(x)=x^{\alpha\{i, j\}}$ for some $\alpha\{i, j\}>0$, then

$$
L\left(v_{1}, \ldots, v_{m}\right)=-\sum_{i=1}^{m} v_{i}+\frac{1}{N} \sum_{\{i, j\} \in E} \frac{\log \left(v_{i}^{\alpha\{i, j\}}+v_{j}^{\alpha\{i, j\}}\right)}{\alpha\{i, j\}}
$$

is a strict Lyapunov function for this variant model.

If furthermore each $\alpha\{i, j\}<1$, then we can argue as in the proof of Theorem 1.4 and conclude that there exists $v$ (depending on $G$ and $\alpha\{i, j\},\{i, j\} \in E$ ) with non-zero entries such that $x(n)$ converges to $v$ almost surely.

\subsection{Hypergraph Based Interactions}

We can similarly define a variant of the model on hypergraphs. Let $G=(V, E)$ be an hypergraph, where $V=[m]$ and $E \subset 2^{V},|E|=N$. Let $x_{1}(n-1), \ldots, x_{m}(n-1)$ be the proportions of balls after step $n-1$. At step $n$, for each hyperedge $e \in E$ add one ball to one of its vertices with probability

$$
\mathbb{P}[i \text { is chosen on hyperedge } e \text { at step } n]=\frac{x_{i}(n-1)}{\sum_{j \in e} x_{j}(n-1)} .
$$

Notice that when $G$ is the trivial hypergraph with only one hyperedge $[m]$, this variant is a Pólya's urn model with balls of $m$ colors. See for instance $\$ 4.2$ of [17].

A special case of this variant was considered in [20]. The authors defined ${ }^{6}$ a model called "Friends II" in a graph $\widetilde{G}=(\{1, \ldots, m\}, \widetilde{E})$. If we define a hypergraph $G=(V, E)$ whose vertices are the edges of $\widetilde{G}$ and whose hyperedges are the sets of neighboring edges in $\widetilde{G}$, i.e. $V=\widetilde{E}$ and $E=\left\{e_{1}, \ldots, e_{m}\right\}$ with $e_{i}=\{\{i, j\} \in \widetilde{E}\}$, then "Friends II" in $\widetilde{G}$ is the same as our variant in the hypergraph $G$.

\footnotetext{
${ }^{6}$ Actually, they only considered the model on complete graphs.
} 
The autonomous ODE of this variant is

$$
\left\{\begin{array}{cc}
\frac{d v_{1}(t)}{d t}=-v_{1}(t)+\frac{1}{N} \sum_{\substack{e \in E \\
1 \in e}} \frac{v_{1}(t)}{\sum_{j \in e} v_{j}(t)} & \vdots \\
\frac{d v_{m}(t)}{d t}=-v_{m}(t)+\frac{1}{N} \sum_{\substack{e \in E \\
m \in e}} \frac{v_{m}(t)}{\sum_{j \in e} v_{j}(t)} .
\end{array}\right.
$$

In this case,

$$
L\left(v_{1}, \ldots, v_{m}\right)=-\sum_{i=1}^{m} v_{i}+\frac{1}{N} \sum_{e \in E} \log \left(\sum_{i \in e} v_{i}\right)
$$

is a strict Lyapunov function.

In addition to the above two variants, one can also consider a model in which the number of balls added at each step is some process possibly depending on the outcome so far.

\section{THE CASE $\alpha=1$ FOR REGULAR BIPARTITE GRAPHS}

This section is of independent interest, and its purpose is to provide a better understanding of the vector field $F$ when $G$ is a finite, $r$-regular, bipartite, connected graph, and $\alpha=1$. We prove that every $v$ in the interior of $\Omega$ is stable in any direction transverse to $\Omega$, by looking at the jacobian matrix

$$
J F=\left[\begin{array}{ccc}
\frac{\partial F_{1}}{\partial v_{1}} & \cdots & \frac{\partial F_{1}}{\partial v_{m}} \\
\vdots & \ddots & \vdots \\
\frac{\partial F_{m}}{\partial v_{1}} & \cdots & \frac{\partial F_{m}}{\partial v_{m}}
\end{array}\right]
$$

of the vector field $F=\left(F_{1}, \ldots, F_{m}\right)$ defined by (3.5). Because $v \in \Omega$ belongs to a line of singularities, 0 is an eigenvalue of $J F(v)$. We prove that

Lemma 10.1. Let $v \in \operatorname{int}(\Omega)$. Any eigenvalue of $J F(v)$ different from 0 has negative real part, and 0 is a simple eigenvalue of $J F(v)$.

Proof. We explicitly calculate the entries $\partial F_{i} / \partial v_{k}$. Let $v \in \Omega$ with $v_{i}=p$ for $i \in A$ and $v_{i}=q$ for $i \in B$. We have five cases:

- $i=k \in A$ :

$$
\frac{\partial F_{i}}{\partial v_{i}}(v)=-1+\frac{1}{N} \sum_{j \sim i} \frac{v_{j}}{\left(v_{i}+v_{j}\right)^{2}}=-1+\frac{m q}{2} .
$$

- $i=k \in B$ : analogously,

$$
\frac{\partial F_{i}}{\partial v_{i}}(v)=-1+\frac{m p}{2}
$$


- $i \sim k$ and $i \in A$ :

$$
\frac{\partial F_{i}}{\partial v_{k}}(v)=-\frac{v_{i}}{N\left(v_{i}+v_{k}\right)^{2}}=-\frac{m p}{2 r} .
$$

- $i \sim k$ and $i \in B$ : analogously,

$$
\frac{\partial F_{i}}{\partial v_{k}}(v)=-\frac{m q}{2 r}
$$

- $i \not k$ : in this case, $\partial F_{i} / \partial v_{k}=0$.

If we label the vertices of $A$ from 1 to $m / 2$, the vertices of $B$ from 1 to $m / 2$, and if we let $M=\left(m_{i j}\right)$ be the $m / 2 \times m / 2$ adjacency matrix of the edges connecting vertices of $A$ to vertices of $B$ (i.e. $m_{i j}=1$ when the $i$-th vertex of $A$ is adjacent to the $j$-th vertex of $B$ ), then $J F(v)$ takes the form

$$
J F(v)=-I+\frac{m}{2 r}\left[\begin{array}{cc}
r q I & -p M \\
-q M^{t} & r p I
\end{array}\right] .
$$

Letting $\mu=p /(p+q)$ and $v=q /(p+q), J F(v)$ can be written as

$$
J F(v)=-I+\frac{1}{r}\left[\begin{array}{cc}
r v I & -\mu M \\
-v M^{t} & r \mu I
\end{array}\right]=:-I+\frac{1}{r} S .
$$

Given a matrix $X$, let $\sigma(X)$ denote its spectrum. By (10.2),

$$
\sigma(J F(v))=\frac{1}{r} \sigma(S)-1
$$

and so it is enough to estimate the set $\sigma(S)$. The lemma will follow once we prove that

a. every element of $\sigma(S)$ is either real or has real part equal to $r / 2$,

b. $r$ is the largest real eigenvalue of $S$, and

c. $r$ is a simple eigenvalue of $S$.

Let's prove (a). Let $\lambda=a+b i \in \sigma(S)$. Because $r \mu, r v<r$, we can assume that $\lambda \neq r \mu, r v$. Note that the matrix

$$
S-\lambda I=\left[\begin{array}{cc}
(r v-\lambda) I & -\mu M \\
-v M^{t} & (r \mu-\lambda) I
\end{array}\right]
$$

is singular if and only if its Schur complement

$$
(r v-\lambda) I-(-\mu M)(r \mu-\lambda)^{-1} I\left(-v M^{t}\right)=\frac{\mu \nu}{r \mu-\lambda}\left[\frac{(r \mu-\lambda)(r v-\lambda)}{\mu \nu} I-M M^{t}\right]
$$

is singular. Because $M M^{t}$ is symmetric, all of its eigenvalues are real and so

$$
\frac{(r \mu-\lambda)(r \nu-\lambda)}{\mu \nu} \in \mathbb{R} \Longrightarrow(r \mu-\lambda)(r \nu-\lambda) \in \mathbb{R} \text {. }
$$


Calculating the imaginary part of $(r \mu-\lambda)(r v-\lambda)$, it follows that

$$
-r b+2 a b=0 \Longrightarrow b=0 \quad \text { or } \quad a=r / 2,
$$

which proves (a).

To prove (b), let $\lambda \in \sigma(S) \cap \mathbb{R}$, say $S x=\lambda x$, where $x=\left(x_{1}, \ldots, x_{m}\right) \in \mathbb{R}^{m} \backslash\{0\}$. Letting $x_{i}=\max \left\{\left|x_{1}\right|, \ldots,\left|x_{m}\right|\right\}$ for $i \in A$, we have

$$
\lambda x_{i}=r v x_{i}-\mu \sum_{j \sim i} x_{j} \leq r v x_{i}+\mu \sum_{j \sim i} x_{i}=r x_{i}
$$

and thus $\lambda \leq r$. The same holds if $i \in B$.

It remains to prove (c). When $\lambda=r$, we have for $i \in A$

$$
r x_{i}=r v x_{i}-\mu \sum_{j \sim i} x_{j} \Longrightarrow x_{i}=-\frac{1}{r} \sum_{j \sim i} x_{j}
$$

and similarly for $i \in B$. Thus the function $h: V \rightarrow \mathbb{R}$ defined by

$$
h(i)=\left\{\begin{aligned}
x_{i} & \text { if } i \in A, \\
-x_{i} & \text { if } i \in B
\end{aligned}\right.
$$

is harmonic in $G$. By the maximum principle, $h$ is constant, and so $r$ is a simple eigenvalue of $S$.

\section{FURTHER QUESTIONS}

This work is part of a program to answer the following

Problem 11.1. Given a finite connected graph $G$ and $\alpha>0$, what is the limiting behavior of $x(n)$ ?

Theorem 1.4 gives a full answer when $\alpha<1$. When $\alpha=1$, it is not clear what to expect, because the properties of the graph should be taken into account. We conjecture the following.

Conjecture. Let $G$ be a finite, connected, not balanced bipartite graph, and let $\alpha=1$. Then there is a single point such that $x(n)$ converges to it almost surely.

When $\alpha>1$ the question remains widely open, even when $G$ is a triangle. The uniform measure is always an equilibrium. When $1<\alpha<4 / 3$, it is stable and thus $x(n)$ converges to it with positive probability. Also, by Remark 8.1, for any $i \in\{1,2,3\}, x_{i}(n)$ converges to zero with positive probability. In general, we think there exists $\alpha_{0}=\alpha_{0}(G)$ such that when $\alpha>\alpha_{0}$

$$
\mathbb{P}\left[\lim _{n \rightarrow \infty} x(n) \in \partial \Delta\right]=1 .
$$

When $G$ is the star graph, item (b) of Theorem 1.5 gives a partial answer to the question.

Now turn attention to the special cases we considered, summarized in Table 1. 
TABLE 1. The Limiting Behavior of $x(n)$.

\begin{tabular}{lccc}
\hline$G$ & Regular Non-Bipartite & Regular Bipartite & Star Graph \\
\hline$\alpha<1$ & uniform measure & uniform measure & $v(m, \alpha)$ of Theorem 1.5 \\
$\alpha=1$ & uniform measure & $\Omega$ & probability $>0$ to \\
$\alpha>1$ & $?$ & $?$ & $(0, \ldots, 0,1)$ and \\
& & & $\left(\frac{1}{m-1}, \ldots, \frac{1}{m-1}, 0\right)$ \\
\hline
\end{tabular}

When $G$ is regular bipartite and $\alpha=1$, Theorem 1.1 says that the limit set of $x(n)$ is contained in $\Omega$. However, we do not know if the limit exists. When the number of vertices is two, the model is the classical Pólya's urn, and in this case it is known that $x(n)$ converges to a point of $\Omega$ almost surely. See e.g. §2.1 of [17].

Problem 11.2. For a general regular bipartite graph and $\alpha=1$, does $x(n)$ converge to a point of $\Omega$ almost surely?

In Section 10 we proved that every point in the interior of $\Omega$ is stable in any direction transverse to $\Omega$.

Problem 11.3. In Theorems 1.1, 1.2 and 1.4, what is the rate of convergence of $x(n)$ to its limit?

This problem is related to the control of the eigenvalues of $J F$ on $\Lambda$, and of quantitative estimates on the precision that $\{x(n)\}_{n \geq 0}$ shadows a real orbit of the ODE associated to $F$. See e.g. $\$ 3.2$ of [17].

Another question of interest is the following

Problem 11.4. What is the correlation between the number of balls in the bins, as a function of $\alpha$ and of the number of steps $n$, e.g. when $G$ is an Euclidean lattice?

Remark 11.5. After the preparation of this manuscript, we learned that the Conjecture and Problem 11.2 were solved affirmatively [5].

\section{ACKNOWLEDGMENTS}

The authors are thankful to Omri Sarig for valuable comments and suggestions. M.B. is supported by Swiss National Foundation, grant 138242. I.B. is the incumbent of the Renee and Jay Weiss Professorial Chair. J.C. is supported by the ISF. During the preparation of this manuscript, Y.L. was a Postdoctoral Fellow at the Weizmann Institute of Science, supported by the ERC, grant 239885. Y.L. is supported by the Brin Fellowship.

\section{REFERENCES}

[1] N. Alon and J. H. Spencer, The probabilistic method, 3rd edition, Wiley-Interscience Series in Discrete Mathematics and Optimization, 2008. 
[2] M. Benaïm, A dynamical system approach to stochastic approximations, SIAM J Control Optim 34 (1996), 437-472.

[3] M. Benaïm, Dynamics of stochastic approximation algorithms, Séminaire de Probabilités XXXIII, 1999, pp. 1-68.

[4] M. Benaïm, O. Raimond, and B. Schapira, Strongly reinforced vertex-reinforced-random-walk on the complete graph, preprint arXiv:1208.6375 (2012).

[5] J. Chen and C. Lucas, Generalized Polya's urn: Convergence at linearity, preprint arXiv:1306.5465 (2013).

[6] F. Chung, S. Handjani, and D. Jungreis, Generalizations of Polya's urn problem, Ann Comb 7 (2003), 141-153.

[7] R. Durrett, Probability: theory and examples, Cambridge Series in Statistical and Probabilistic Mathematics, Cambridge University Press, Cambridge, 2010.

[8] B. M. Hill, D. Lane, and W. Sudderth, A strong law for some generalized urn processes, Ann Probab 8 (1980), 214-226.

[9] M. O. Jackson, Social and economic networks, Princeton University Press, Princeton, NJ, 2008.

[10] J. Kiefer and J. Wolfowitz, Stochastic estimation of the maximum of a regression function, Ann Math Stat 23 (1952), 462-466.

[11] H. J. Kushner and D. S. Clark, Stochastic approximation methods for constrained and unconstrained systems, Appl Math Sci, Vol. 26, Springer-Verlag, New York-Berlin, 1978.

[12] M. Launay, Interacting urn models, arXiv:1101.1410 (2011).

[13] L. Ljung, Analysis of recursive stochastic algorithms, IEEE Trans Automatic Control AC-22 (1977), 551-575.

[14] M. Marsili and A. Valleriani, Self organization of interacting Polya urns, Eur Phys J B 3 (1998), 417-420.

[15] H. Ohtsuki, C. Hauert, E. Lieberman, and M. A. Nowak, A simple rule for the evolution of cooperation on graphs and social networks, Nature 441 (2006), 502-505.

[16] R. Pemantle, Vertex-reinforced random walk, Probab Theory Related Fields 92 (1992), 117-136.

[17] R. Pemantle, A survey of random processes with reinforcement, Probab Surv 4 (2007), 1-79.

[18] H. Robbins and S. Monro, A stochastic approximation method, Ann Math Stat 22 (1951), 400-407.

[19] F. C. Santos, J. M. Pacheco, and T. Lenaerts, Evolutionary dynamics of social dilemmas in structured heterogeneous populations, Proc Nat Acad Sci USA 103 (2006), 3490-3494.

[20] B. Skyrms and R. Pemantle, A dynamic model of social network formation, Proc Nat Acad Sci USA 97 (2000), 9340-9346. 